\title{
Bisdemethoxycurcumin protects endothelial cells against $t$-BHP-induced cell damage by regulating the phosphorylation level of ERK1/2 and Akt
}

\author{
YING-BO LI, JIAN-LI GAO, SIMON MING-YUEN LEE, QING-WEN ZHANG, \\ PUI-MAN HOI and YI-TAO WANG
}

\begin{abstract}
Institute of Chinese Medical Sciences, University of Macau, Av. Padre Tomás Pereira S.J., Taipa, Macao, P.R. China
\end{abstract}
Received June 9, 2010; Accepted September 3, 2010

DOI: $10.3892 / \mathrm{ijmm} .2010 .578$

\begin{abstract}
Curcuminoids are the major active components extracted from Curcuma longa and are well known for their antioxidant effects. Previous studies have reported that the antioxidant properties of curcuminoids are mainly attributed to their free radical scavenging abilities. However, whether there are other mechanisms besides the non-enzymatic process and how they are involved, still remains unknown. In the present study, we explored the protective effects of bisdemethoxycurcumin (Cur3) against tert-butyl hydroperoxide $(t$-BHP)-induced cytotoxicity in human umbilical vein endothelial cells (HUVECs), focusing on the effect of Cur3 on the regulation of the phosphatidylinositol 3-kinase (PI3K)/Akt and the mitogen-activated protein kinase (MAPK) pathways. The pre-treatment with Cur3 inhibited $t$-BHPinduced cell damage dose-dependently, which was evident by the increased cell viability and the corresponding decrease in lactate dehydrogenase release. The pre-treatment with Cur3 also attenuated $t$-BHP-induced cell morphological changes and apoptosis. MAPKs, including p38, c-Jun Nterminal kinase (JNK), extracellular signal-regulated protein kinase 1/2 (ERK1/2), as well as PI3K/Akt have been reported to be involved in proliferation, apoptosis and differentiation under various stress stimulations. The pre-treatment with Cur3 decreased $t$-BHP-induced ERK1/2 phosphorylation and increased $t$-BHP-induced Akt phosporylation but did not affect the phosphorylation of $\mathrm{p} 38$ or JNK. In addition, the Cur3-induced increase in cell viability was attenuated by the treatment with wortmannin or LY294002, the upstream inhibitors of Akt, and was enhanced by the treatment with 2-[2'-amino-3'-methoxyphenyl]-oxanaphthalen-4-one (PD98059), an upstream inhibitor of ERK1/2. These results
\end{abstract}

Correspondence to: Professor Yi-Tao Wang or Dr Pui-Man Hoi, Institute of Chinese Medical Sciences, University of Macau, Av. Padre Tomás Pereira S.J., Taipa, Macao, P.R. China

E-mail: ytwang@umac.mo

E-mail:maghoi@umac.mo

Key words: curcuminoids, oxidative stress, endothelial cells, mitogen-activated protein kinase, Akt suggest that the ERK1/2 and PI3K/Akt signaling pathways could be involved in the protective effects of Cur3 against $t$-BHP-induced damage in HUVECs.

\section{Introduction}

Curcuma longa has been used in traditional medicine to treat mild or moderate human diseases in India, China and other Asian countries for centuries (1-3). Over the years, it has sparked increasing scientific curiosity due to its broad range of potent pharmacological effects and its almost non-existent toxicity even at high doses (4). Curcuminoids, including curcumin (Cur1), demethoxycurcumin (Cur2) and bisdemethoxycurcumin (Cur3), are the main active components extracted from the rhizome of Curcuma longa, and have been demonstrated to exert various therapeutic, including antiinflammatory, anti-carcinogenic, anti-infectious, as well as neuro- and cardioprotective effects $(1,4-7)$.

It is known that the structure and functional integrity of endothelial cells play very important roles in the maintenance of healthy vessel walls and circulatory functions (8). Oxidative stress is regarded as one of the major causes of endothelial cell damage (9), and thereby contributes to most major cardiovascular diseases, such as atherosclerosis, hypertension and thrombosis (10). Curcuminoids are well known for their potent protective effects against oxidative stress, and have therefore been suggested as attractive therapeutic agents for the prevention and treatment of free radical-related diseases, including cardiovascular diseases. However, even though data obtained from laboratory experiments and clinical trials have shown promising antioxidant activities of curcuminoids, the underlying mechanisms have yet to be fully clarified. In previous studies, the protective effects of curcuminoids have mainly been attributed to the free radical scavenging ability of the phenolic hydroxyl group in their molecular structures (11-13). However, other studies have suggested that more complex mechanisms are involved. It has been demonstrated that curcuminoids can induce the expression of heme oxygenase-1 (HO-1), which contributed to their cytoprotective effects in response to oxidative injuries. This effect was associated with increased Nrf2 binding to the resident ho- 1 antioxidant-responsive element as well as with the activation of mitogen-activated protein kinases (MAPKs) $(14,15)$. Moreover, curcumin has been reported to increase the 
expressions of other antioxidant enzymes such as superoxide dismutase, glutathione S-transferase and aldo-keto reductase, and the phosphatidylinositol 3-kinase (PI3K)/Akt and the p38 MAPK pathways have also been shown to be involved in the regulations of some of these enzymes $(16,17)$.

In view of the evidence that the MAPK and Akt pathways are two of the most commonly involved signal transduction pathways in response to oxidative challenges by the regulation of cell proliferation, apoptosis and death $(18,19)$, we postulated that the MAPK and PI3K/Akt pathways could also play significant roles in the underlying mechanisms of the protective effects of curcuminoids against oxidative injuries. In order to test this hypothesis, exogenous tert-butyl hydroperoxide $(t$-BHP) was used to induce endothelial cell damage. The effects of curcuminoids against oxidative stress were examined and possible pathways were analyzed. The results suggest that Cur3 exhibited a potent protective effect against $t$-BHP-induced cell damage in human umbilical vein endothelial cells (HUVECs) which could be mediated through the regulation of the extracellular signal-regulated protein kinase 1/2 (ERK1/2) and PI3K/Akt pathways.

\section{Materials and methods}

Materials. Kaighn's modification of Ham's F12 medium (F-12K), fetal bovine serum (FBS), phosphate buffered saline (PBS), penicillin, streptomycin, and $0.25 \%(\mathrm{w} / \mathrm{v})$ trypsin/1 mM EDTA, were purchased from Invitrogen (Carlsbad, CA, USA). Endothelial cell growth supplement, heparin, gelatin and $t$-BHP solution $70 \%$ in $\mathrm{H}_{2} \mathrm{O}$ were supplied by Sigma (St. Louis, MO, USA). MTT was purchased from USB (OH, USA). The Cytotoxicity Detection Kit was purchased from Roche Applied Science (Germany). Hoechst 33342 dye was purchased from Molecular Probe (Eugene, OR, USA). All antibodies were purchased from Cell Signaling Technology (Beverly, MA, USA). PD98059, LY294002 and wortmannin were purchased from Merck (Germany). RIPA lysis buffer, phenylmethylsulfonyl fluoride (PMSF), protease inhibitor cocktail and the $\mathrm{BCA}^{\mathrm{TM}}$ Protein Assay kit were purchased from Pierce Biotechnology (Rockford, IL, USA). The ECL advanced Western blotting detection kit was purchased from Amersham (UK)

Drugs. Pure curcumin (MW 368), demethoxycurcumin (MW 338) and bisdemethoxycurcumin (MW 308) were separated from Curcuma longa (turmeric). Their structures were deduced on the basis of their physicochemical properties and spectral data (Fig. 1), and the purity of the compounds was $>98 \%$. The purity was verified by HPLC-DAD.

Cell culture. HUVECs were purchased from ATCC (Manassas, USA), and maintained at $37^{\circ} \mathrm{C}$ and $5 \% \mathrm{CO}_{2}$ in F-12K medium supplemented with $2 \mathrm{mM}$ L-glutamine, $1.5 \mathrm{~g} / 1$ sodium bicarbonate, $30 \mathrm{mg} / \mathrm{l}$ ECGS, 10\% (v/v) FBS, $100 \mathrm{mg} / \mathrm{l}$ heparin, $100 \mathrm{U} / \mathrm{ml}$ penicillin and $100 \mu \mathrm{g} / \mathrm{ml}$ streptomycin. All experiments were performed with cells between passages 2 to 5 .

Cell viability assays. HUVECs were seeded at $5 \times 10^{3}$ cells/ well in 96-well plates. After $24 \mathrm{~h}$ of pre-treatment with

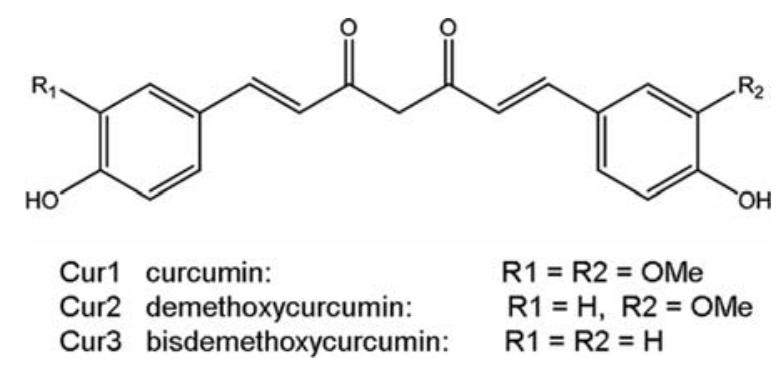

Figure 1. The chemical structures of curcumin, demethoxycurcumin and bisdemethoxycurcumin.

curcuminoids and $1 \mathrm{~h} t$-BHP exposure, cell viability was evaluated by MTT and lactate dehydrogenase (LDH) assays. In the MTT assay, the HUVECs were incubated with $100 \mu 1$ F-12K medium containing $0.5 \mathrm{mg} / \mathrm{ml} \mathrm{MTT}$. After $4 \mathrm{~h}$ incubation at $37^{\circ} \mathrm{C}$, the cell supernatants were discarded, the MTT crystals were dissolved in $100 \mu 1 \mathrm{DMSO}$ and the absorbance was measured at $570 \mathrm{~nm}$ using a multilabel counter (Perkin Elmer, 1420 Multilabel Counter Victor3, Wellesley, MA, USA). In the LDH assay, cell injury was assessed by measuring the amount of LDH released into the medium from damaged cells. LDH activity in the cell culture supernatants was determined using the Cytotoxicity Detection Kit according to the manufacturer's instructions. The release of LDH was measured at $490 \mathrm{~nm}$ with $690 \mathrm{~nm}$ as the reference wavelength.

Hoechst labeling. Hoechst 33342 staining was used to observe the chromosome condensation and morphological changes of the cells. HUVECs were cultured in 24-well plates. The cells were treated with $30 \mu \mathrm{M}$ Cur3 for $24 \mathrm{~h}$, followed by the stimulation of $300 \mu \mathrm{M} t$-BHP for $1-2 \mathrm{~h}$. The cells were fixed with $4 \%$ paraformaldehyde and washed with PBS, and then stained with Hoechst $33342(10 \mu \mathrm{g} / \mathrm{ml})$ with RNase $(5 \mu \mathrm{g} / \mathrm{ml})$ for $30 \mathrm{~min}$ at room temperature. After 3 washes with PBS, stained cells were visualized under UV illumination using a fluorescent microscope (Carl Zeiss, Axiovert 200, USA).

Western blot analysis. Protein was extracted using RIPA lysis buffer with $1 \% \mathrm{PMSF}$ and $1 \%$ protease inhibitor. Lysates were centrifuged at $12,000 \mathrm{x}$ g for $20 \mathrm{~min}$ at $4^{\circ} \mathrm{C}$ and the supernatant was collected. Total protein concentrations were determined using the $\mathrm{BCA}^{\mathrm{TM}}$ Protein Assay kit. Supernatants containing $40 \mu \mathrm{g}$ of protein/lane were separated by $12 \%$ SDS-PAGE gels. After electrophoresis, the separated proteins were electrically transferred onto polyvinylidene difluoride membranes. The membrane was probed with a primary antibody followed by a second antibody and visualized using an ECL advanced Western blotting detection kit. Photos of protein bands were taken using the Molecular Imager ChemiDoc XRS (BioRad, USA). Densitometric measurements of the band intensities on the Western blots were performed using Quantity One Software.

Statistical analysis. Results were presented as the means \pm SD of at least 3 independent experiments. Data were analyzed by 

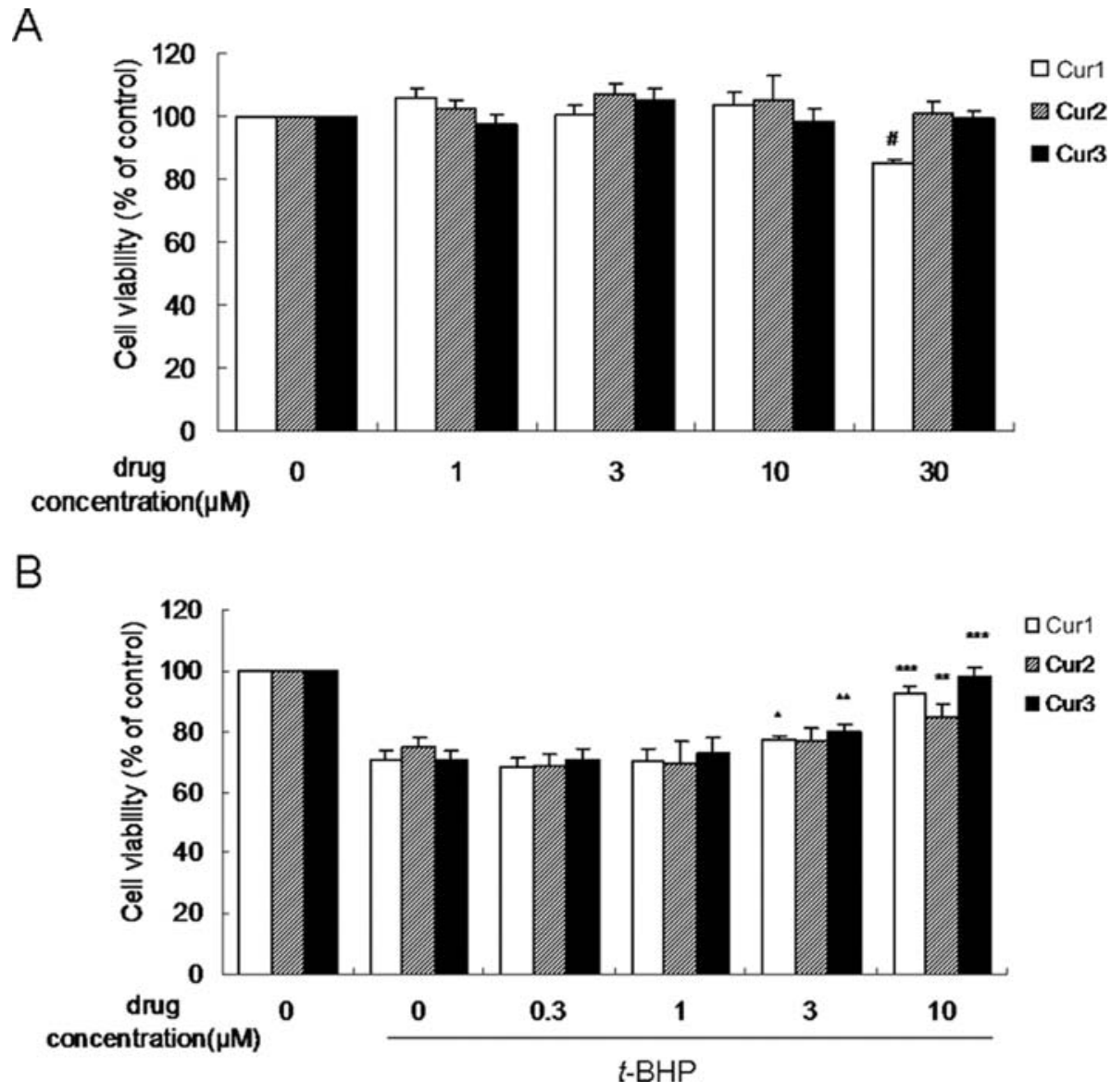

Figure 2. Cytotoxicity and protective effects of curcuminoids against $t$-BHP-induced cell damage in HUVECs. (A) Effects of curcuminoids on the viability of HUVECs. The cells were exposed to various concentrations of Cur1, 2 or $3(1-30 \mu \mathrm{M})$ for $24 \mathrm{~h}$, then the cell viability was determined by MTT assay. (B) Protective effects of curcuminoids against $t$-BHP-induced cell damage. The cells were pre-treated with or without Cur1, 2 and 3 for $24 \mathrm{~h}$, followed by $300 \mu \mathrm{M}$ $t$-BHP stimulation for $1 \mathrm{~h}$. The results are presented as a percentage of the control. ${ }^{*} \mathrm{P}<0.05$ compared to the untreated controls, ${ }^{*} \mathrm{P}<0.05$, ${ }^{* *} \mathrm{P}<0.01$ and ${ }^{* * *} \mathrm{P}<0.001$ compared to the $t$-BHP alone group. The data shown here are the means \pm SD of 3 independent experiments.

the Student's t-test. A value of $\mathrm{P}<0.05$ was considered to be statistically significant.

\section{Results}

Curcuminoids protected HUVECs against t-BHP-induced cytotoxicity. We first examined and compared the cytotoxicity and protective effects of 3 curcuminoids, Cur 1, 2 and 3 , against $t$-BHP-induced cell damage. HUVECs were treated with different doses of Cur1, 2 or 3 for $24 \mathrm{~h}$ followed by MTT assay. The results show that Cur2 and 3 did not exhibit any cytotoxicity up to a dose of $30 \mu \mathrm{M}$, while Cur 1 slightly decreased the cell viability at the highest concentration used (30 $\mu \mathrm{M}, 24 \mathrm{~h}$ ) (Fig. 2A). The protective effects of these 3 curcuminoids were further explored by exposing HUVECs to $t$-BHP $(300 \mu \mathrm{M})$ for $1 \mathrm{~h}$ with or without the pre-treatment $(24 \mathrm{~h})$ with curcuminoids. As shown in Fig. 2B, the pretreatments with Cur1 and 3 attenuated $t$-BHP-induced cell damage in a dose-dependent manner and they reached their maximal effects at a concentration of $10 \mu \mathrm{M}(\mathrm{P}<0.001)$. The pre-treatment with Cur2 also modestly protected the cells against the oxidative damage elicited by $t$-BHP in a dosedependent manner with a maximal effect at a dose of $10 \mu \mathrm{M}$ $(\mathrm{P}<0.01)$. Considering that Cur3 exhibited the best perfor- mance among the 3 curcuminoids, it was chosen for further study.

Cur3 protected HUVECs against t-BHP-induced cell death and apoptosis. In order to further verify the cellular protective effect of Cur3, the LDH assay was performed. The release of LDH was dramatically increased to $141.01 \%$ of the control when the cells were challenged with $t$-BHP-induced oxidative stress. The pre-treatment with Cur3 decreased LDH release dose-dependently with a maximal effect at a dose of $30 \mu \mathrm{M}$ (116.74\% of the control). The effect of the Cur3 pre-treatment on $t$-BHP-induced morphological changes and apoptosis in HUVECs was also examined. As shown in Fig. 3, $t$-BHP induced various morphological changes, including the loss of cell attachment, cell shrinkage, nucleus pycnosis, and the formation of crescent-shaped condensation or apoptotic bodies which were not observed in the control group. Fig. 3D shows that such morphological changes and apoptotic features were reduced significantly when the cells were pre-treated with $30 \mu \mathrm{M}$ of Cur3.

t-BHP treatment activated MAPK [c-Jun N-terminal kinase (JNK), p38 and ERK1/2] and Akt pathways. In order to investigate whether the MAPK and Akt pathways were 

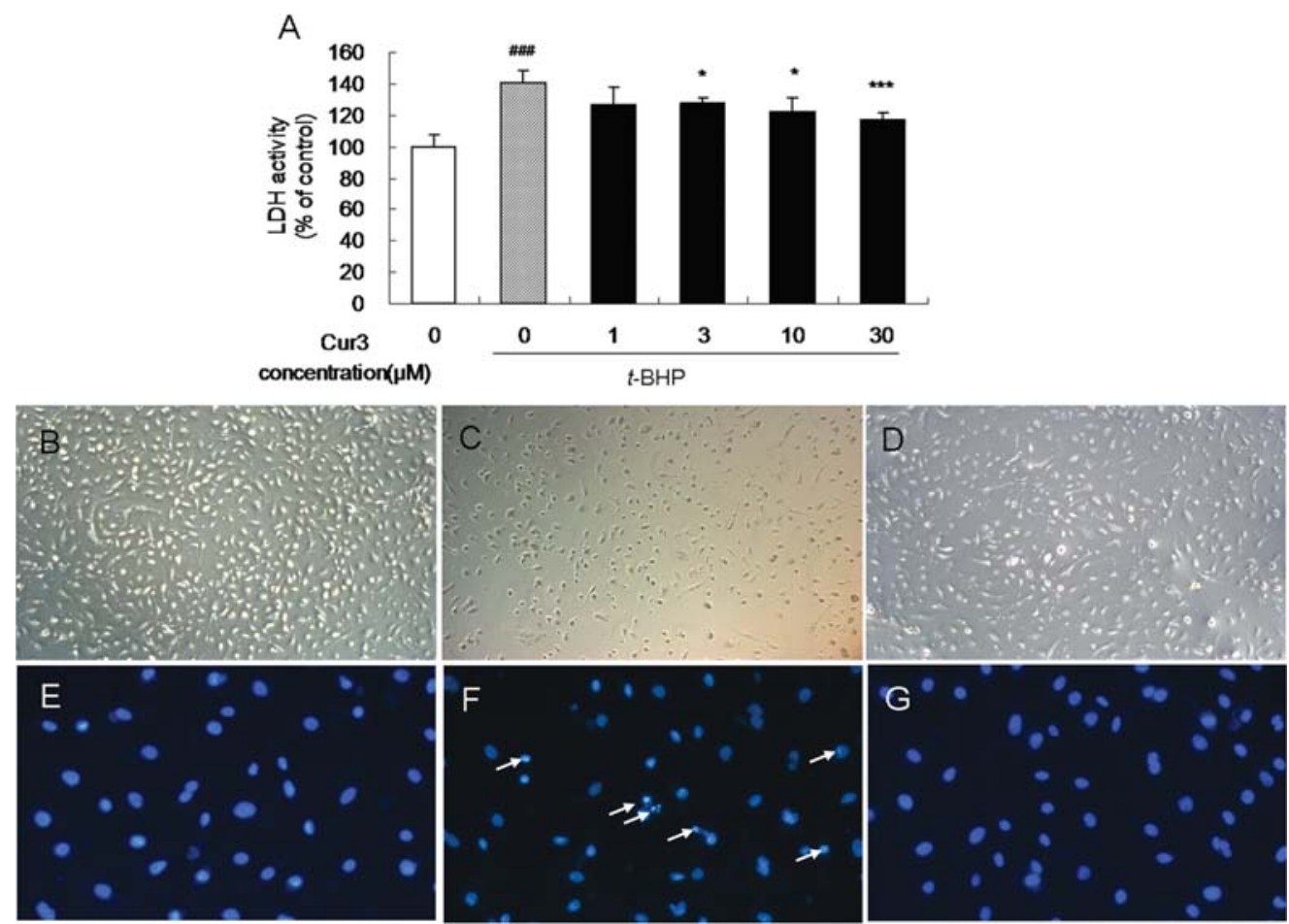

Figure 3. Protective effect of Cur3 against $t$-BHP-induced cell damage in HUVECs. (A) Effect of Cur3 pre-treatment on LDH leakage induced by $t$-BHP in HUVECs. Cells were treated with or without various concentrations of Cur3 for $24 \mathrm{~h}$, followed by $t$-BHP stimulation for $1 \mathrm{~h}$. LDH release was then measured as described in Materials and methods. ${ }^{\# \#} \mathrm{P}<0.001$ compared to the untreated control group. ${ }^{*} \mathrm{P}<0.05,{ }^{* * *} \mathrm{P}<0.001$ compared to $t$-BHP alone group. (B-D) Representative images of HUVECs. (E-G) Representative images of Hoechst 33342 staining. Chromatin condensation and DNA fragments are indicated by the white arrows. (B and E) Untreated control. (C and F) Cells treated with $t$-BHP $300 \mu \mathrm{M}$ for 1-2 $\mathrm{h}$. (D and G) Cells pre-treated with $30 \mu \mathrm{M}$ Cur3 for $24 \mathrm{~h}$ before being exposed to $t$-BHP.

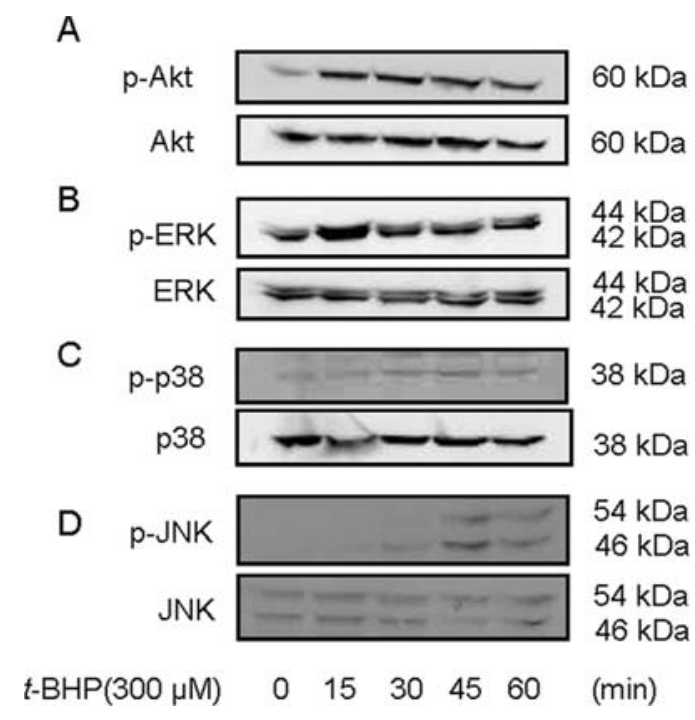

Figure 4. Phosphorylation of Akt, ERK1/2, p38 and JNK induced by $t$-BHP. Cells were treated with $t$-BHP $(300 \mu \mathrm{M})$ and harvested at the times indicated for Western blot analysis. Antibodies against (A) phospho-Akt (Ser473) and Akt, (B) phospho-ERK1/2 (Thr202/Tyr204) and ERK1/2, (C) phospho-p38 (Thr180/Tyr182) and p38, and (D) phospho-JNK (Thr183/Tyr185) and JNK, were applied as described in Materials and methods.

involved in the $t$-BHP-induced oxidative injury, the phosphorylation levels of JNK (Thr183/Tyr185), p38 (Thr180/ Tyr182), ERK1/2 (Thr202/Tyr204) and Akt (Ser473) were examined by Western blot analysis. Fig. 4 shows that $t$-BHP treatments $(300 \mu \mathrm{M})$ triggered rapid activations of JNK, p38, ERK1/2 and Akt in HUVECs with no significant change in the total protein levels. The maximal levels of phosphorylation for Akt and ERK1/2 were observed at $15 \mathrm{~min}$, while those for p38 and JNK, at $45 \mathrm{~min}$.

The pre-treatment with Cur3 modulated t-BHP-induced ERK1/2 and Akt activation. The pre-treatment with Cur3 modulated the $t$-BHP-induced MAPK and Akt activation. HUVECs were first pre-treated with Cur3 (0.3-30 $\mu \mathrm{M})$ for $24 \mathrm{~h}$, followed by $300 \mu \mathrm{M} t$-BHP. Based on the timeresponse results described earlier (Fig. 4), the cells were exposed to $t$-BHP for $15 \mathrm{~min}$ to evaluate the effects on the regulation of ERK1/2 and Akt, and 45 min for JNK and p38, respectively (Fig. 5). The results show that the pre-treatment with Cur3 for $24 \mathrm{~h}$ down-regulated the activation of ERK1/2. On the contrary, the phosphorylation level of Akt was increased in a dose-dependent manner. However, the Cur3 pre-treatment did not produce any significant modulation on the $t$-BHP-induced activations of $\mathrm{p} 38$ or JNK.

t-BHP-induced cytotoxicity of HUVECs was attenuated by PD98059 and enhanced by wortmannin or LY294002. Specific MEK1 (PD98059) and PI3K inhibitors (wortmannin and LY294002) were used to further clarify whether the $t$-BHPinduced cytotoxicity was mediated through the ERK1/2 or PI3K/Akt signaling pathways. The HUVECs were exposed to PD98059 $(10 \mu \mathrm{M})$, LY294002 $(20 \mu \mathrm{M})$ or wortmannin $(20 \mu \mathrm{M})$ for $1 \mathrm{~h}$ before the treatment with $t$-BHP. The cells 

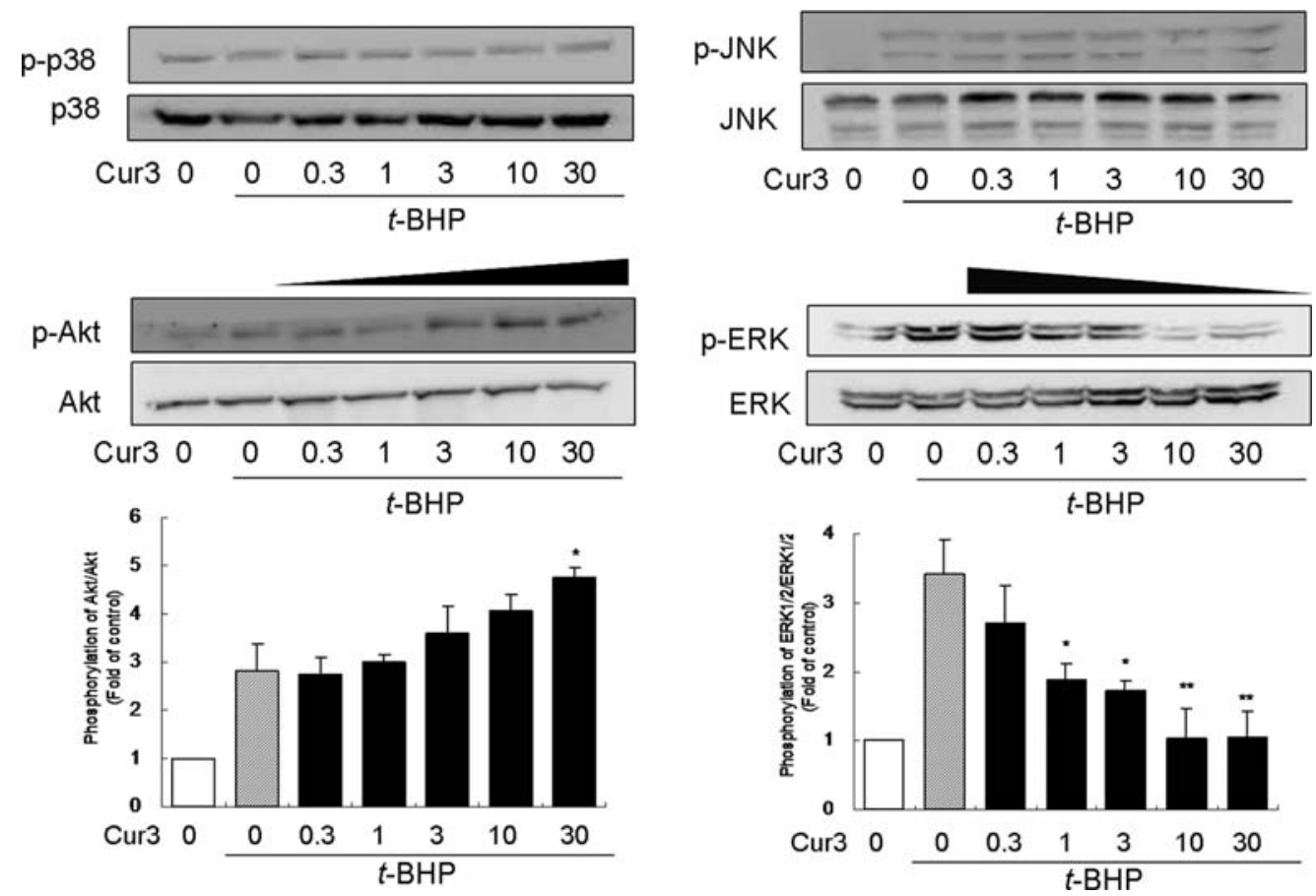

Figure 5. Effects of Cur3 on $t$-BHP-induced phosphorylation of Akt, ERK1/2, p38 and JNK. Cells were pre-treated with various concentrations of Cur3 for $24 \mathrm{~h}$, and then stimulated with $t$-BHP $(300 \mu \mathrm{M})$ for 15 min for the determination of ERK1/2 and Akt activities, or for 45 min for the determination of p38 and JNK activities. Representative blots are shown. At least 3 independent experiments were done for each investigation.

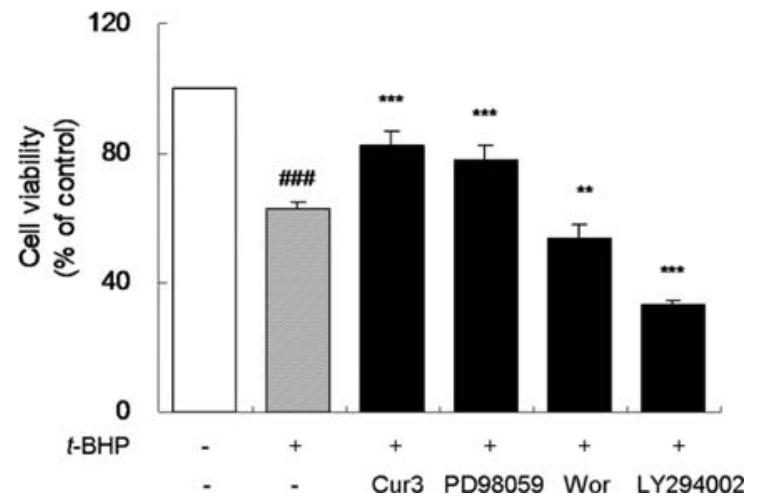

Figure 6. Effects of PD98059, wortmannin and LY294002 on $t$-BHP-induced cell damage. The cells were pre-treated with Cur3 $(30 \mu \mathrm{M}, 24 \mathrm{~h})$ PD98059 $(10 \mu \mathrm{M}, 1 \mathrm{~h})$, wortmannin $(20 \mu \mathrm{M}, 1 \mathrm{~h})$ or LY294002 $(20 \mu \mathrm{M}, 1 \mathrm{~h})$, followed by the stimulation of $300 \mu \mathrm{M} t$-BHP for $1 \mathrm{~h}$. Cell viability was measured by MTT assay. The results are presented as a percentage of the control. ${ }^{\# \# \#} \mathrm{P}<0.001$ compared to the untreated controls, ${ }^{*} \mathrm{P}<0.05,{ }^{* *} \mathrm{P}<0.01$ and ${ }^{* * *} \mathrm{P}<0.001$ compared to the $t$-BHP alone group. The data shown here are the means $\pm \mathrm{SD}$ of 3 independent experiments.

were then further incubated for $1 \mathrm{~h}$ in the presence of $300 \mu \mathrm{M}$ $t$-BHP followed by subsequent MTT assay. PD98059 (10 $\mu \mathrm{M}$, $1 \mathrm{~h})$ blocked the phosphorylation of ERK1/2 (Thr202/Tyr204), while wortmannin $(20 \mu \mathrm{M}, 1 \mathrm{~h})$ or LY294002 $(20 \mu \mathrm{M}, 1 \mathrm{~h})$ blocked the phosphorylation of Akt (Ser473). As shown in Fig. 6, the decrease in cell viability caused by $t$-BHP was markly attenuated by PD98059. In contrast, wortmannin and LY294002 enhanced the $t$-BHP-induced cell damage. On the whole, these results suggest that the protective effect of Cur3 against $t$-BHP-induced damage in HUVECs could be mediated through the ERK1/2 and PI3K/Akt pathways.

\section{Discussion}

In the present study, we explored the protective effects of curcuminoids against $t$-BHP-induced damage in HUVECs. $t$-BHP, a reactive hydroperoxide produced from lipid peroxidation, has been shown to be a major player in the activation of the redox-sensitive transcription factors (20), and therefore has frequently been used in the investigation of cellular alterations resulting from free radical actions in various cell types, such as hepatocyte (21), auditory (22) retinal pigmented epithelial (23), and endothelial cells (20). Consistent with previous reports, $t$-BHP induced cell damage and even cell death, which was evident by the decreased cell viability and increased LDH release. Cur1, 2 and 3, which are 3 active curcuminoids extracted from the rhizome of Curcuma longa, significantly protected HUVECs from $t$-BHP-induced oxidative damage. Among them, Cur3 exerted the best protective effect with the lowest cytotoxicity. In addition, Cur3 attenuated $t$-BHP-induced cell morphological changes and apoptosis, such as the loss of cell attachment, cell shrinkage, nucleus pycnosis, and the formation of crescent-shaped or apoptotic bodies in HUVECs. Therefore, Cur3 was selected for further study in order to explore the underlying mechanisms in further detail.

Oxidative stress triggered various kinds of signaling pathways, among which MAPK and Akt have been proven to play important roles in damages caused by ROS (24). Our results show that the exposure of HUVECs to $t$-BHP rapidly activates JNK, p38, ERK1/2 and Akt without changing the total protein levels. Furthermore, it was found that Cur3 dosedependently decreased the ERK $1 / 2$, whereas it increased the Akt phosphorylation level. These results indicate the possibility of the involvement of ERK1/2 and Akt in the 
protective effects of Cur3 against $t$-BHP-induced cell damage in HUVECs.

The ERK1/2 pathway, as a subfamily of MAPK whose function and regulation are evolutionarily conserved, has been reported to be involved in many cellular processes such as growth, proliferation, differentiation and death. In addition, the ERK $1 / 2$ pathway has been reported to be rapidly activated in response to various cellular stimuli, including oxidative stress. Our results confirm that $t$-BHP increased the phosphorylation level of ERK1/2 which peaked at $15 \mathrm{~min}$. In order to clarify the role of ERK1/2 in the process of $t$-BHPinduced damage, PD98059, which is a specific inhibitor of the upstream factor of ERK, was used. The results demonstrate that when the ERK pathway was blocked by the inhibitor, the cell damage caused by $t$-BHP stimulation, was attenuated. This observation is not consistent with previous studies which generally consider ERK $1 / 2$ to be a cell survival signal in the process of ROS-triggered apoptosis. However, other evidence has suggested that the activation of ERK1/2 also mediates cell damage (25). It has been reported that blocking the ERK pathway by using MEK1 inhibitors rescues cells from injury induced by various stimuli $(26,27)$. This observation was further confirmed by the observation that the transient transfection of cells with constitutively active MEK1 increased the cisplatin-induced apoptosis, whereas the transfection with a dominant-negative mutant of MEK1, decreased it (28). Additionally, the inhibition of ERK activation has been implicated in the protective effects of drugs such as salvianolic acid B against hydrogen peroxideinduced endothelial cell apoptosis (29). These results suggest that the decrease in the phosphorylation level of ERK1/2 could be beneficial to cell survival in some cell types and organs under certain conditions.

Growing evidence indicates that the Akt signaling pathway plays a crucial role in cell survival in response to oxidative stress. Several downstream targets of Akt have been found to be involved in the regulation of cell apoptosis, DNA repair, stress resistance and metabolism. For example, it has been reported that Akt can phosphorylate the Bcl-2 family member, BAD, both in vivo and in vitro, and promotes cell survival and suppresses BAD-mediated cell death (30). NF-кB and caspase- 9 have also been identified as functional targets of Akt (31). Our results show that the $t$-BHP-induced cell death of HUVECs was accompanied with an increased level of Akt phosphorylation. However, the Akt phosphorylation reached its maximal level at $15 \mathrm{~min}$ after $t$-BHP exposure, but was not maintained and started to decline soon afterwards. This could be explained by the effect of stress response which occurred instantly and only temporarily as the first call of defense of the cells against injuries. Nevertheless, our results clearly show that the pre-treatment with Cur3 significantly prevented cell loss and dose-dependently increased the level of phosphorylated Akt when the cells were challenged with $t$-BHP, suggesting that Cur3 produced its protective effects through the up-regulation of Akt activation. Furthermore, the $t$-BHP-induced cell damage was enhanced by blocking the PI3K/Akt pathway with wortammin or LY294002. It has been reported that cytotoxicity induced by a variety of apoptotic stimuli can be attenuated effectively by the constitutive activation of Akt, while the transfection with dominant-negative Akt alleles abrogates the ability of various growth factors to promote cell survival (32-34). Consistent with previous findings, it is evident that the $\mathrm{PI} 3 \mathrm{~K} / \mathrm{Akt}$ pathway is essential for cell survival and apoptosis, and that the up-regulation of Akt activation could be one of the under-lying mechanisms that contribute to the protective effects of Cur3.

In summary, the present study demonstrates that Cur3 protects $t$-BHP-induced endothelial cell damage by downregulating the activation of ERK1/2 and up-regulating the phosphorylation of Akt. This study provides experimental evidence to support the notion that curcuminoids could have therapeutic and protective applications in free radical-related cardiovascular diseases.

\section{Acknowledgements}

The current study was supported by the University of Macau (Project no. RG084/07-08S/WYT/ICMS) and the Macao Science and Technology Development Fund (no. 029/2007/A2).

\section{References}

1. Aggarwal BB and Harikumar KB: Potential therapeutic effects of curcumin, the anti-inflammatory agent, against neurodegenerative, cardiovascular, pulmonary, metabolic, autoimmune and neoplastic diseases. Int J Biochem Cell Biol 41: 40-59, 2009.

2. Aggarwal BB, Sundaram C, Malani N and Ichikawa H: Curcumin: the Indian solid gold. Adv Exp Med Biol 595: 1-75, 2007.

3. Maheshwari RK, Singh AK, Gaddipati J and Srimal RC: Multiple biological activities of curcumin: a short review. Life Sci 78: 2081-2087, 2006.

4. Miriyala S, Panchatcharam M and Rengarajulu P: Cardioprotective effects of curcumin. Adv Exp Med Biol 595: 359$377,2007$.

5. Thangapazham RL, Sharma A and Maheshwari RK: Multiple molecular targets in cancer chemoprevention by curcumin. AAPS J 8: E443-E449, 2006.

6. Wang R, Li YB, Li YH, Xu Y, Wu HL and Li XJ: Curcumin protects against glutamate excitotoxicity in rat cerebral cortical neurons by increasing brain-derived neurotrophic factor level and activating TrkB. Brain Res 1210: 84-91, 2008.

7. Sandur SK, Ichikawa H, Pandey MK, et al: Role of pro-oxidants and antioxidants in the anti-inflammatory and apoptotic effects of curcumin (diferuloylmethane). Free Radic Biol Med 43: 568-580, 2007.

8. Sumpio BE, Riley JT and Dardik A: Cells in focus: endothelial cell. Int J Biochem Cell Biol 34: 1508-1512, 2002.

9. Wickens AP: Ageing and the free radical theory. Respir Physiol 128: 379-391, 2001.

10. Cines DB, Pollak ES, Buck CA, et al: Endothelial cells in physiology and in the pathophysiology of vascular disorders. Blood 91: 3527-3561, 1998.

11. Calabrese V, Bates TE, Mancuso C, et al: Curcumin and the cellular stress response in free radical-related diseases. Mol Nutr Food Res 52: 1062-1073, 2008.

12. Ruby AJ, Kuttan G, Babu KD, Rajasekharan KN and Kuttan R: Anti-tumour and antioxidant activity of natural curcuminoids. Cancer Lett 94: 79-83, 1995.

13. Joe B and Lokesh BR: Role of capsaicin, curcumin and dietary n-3 fatty acids in lowering the generation of reactive oxygen species in rat peritoneal macrophages. Biochim Biophys Acta 1224: 255-263, 1994.

14. Balogun E, Hoque M, Gong P, et al: Curcumin activates the haem oxygenase- 1 gene via regulation of Nrf2 and the antioxidant-responsive element. Biochem J 371: 887-895, 2003.

15. Jeong GS, Oh GS, Pae HO, et al: Comparative effects of curcuminoids on endothelial heme oxygenase-1 expression: ortho-methoxy groups are essential to enhance heme oxygenase activity and protection. Exp Mol Med 38: 393-400, 2006. 
16. Kang ES, Woo IS, Kim HJ, et al: Up-regulation of aldose reductase expression mediated by phosphatidylinositol 3-kinase/ Akt and Nrf2 is involved in the protective effect of curcumin against oxidative damage. Free Radic Biol Med 43: 535-545, 2007.

17. Motterlini R, Foresti R, Bassi R and Green CJ: Curcumin, an antioxidant and anti-inflammatory agent, induces heme oxygenase- 1 and protects endothelial cells against oxidative stress. Free Radic Biol Med 28: 1303-1312, 2000.

18. Owuor ED and Kong AN: Antioxidants and oxidants regulated signal transduction pathways. Biochem Pharmacol 64: 765-770, 2002.

19. Feuerstein GZ and Young PR: Apoptosis in cardiac diseases: stress- and mitogen-activated signaling pathways. Cardiovasc Res 45: 560-569, 2000.

20. Lee JY, Yu BP and Chung HY: Activation mechanisms of endothelial NF-kappaB, IKK, and MAP kinase by tert-butyl hydroperoxide. Free Radic Res 39: 399-409, 2005.

21. Martin C, Martinez R, Navarro R, Ruiz-Sanz JI, Lacort M and Ruiz-Larrea MB: tert-Butyl hydroperoxide-induced lipid signaling in hepatocytes: involvement of glutathione and free radicals. Biochem Pharmacol 62: 705-712, 2001.

22. Choi BM and Kim BR: Upregulation of heme oxygenase-1 by brazilin via the phosphatidylinositol 3-kinase/Akt and ERK pathways and its protective effect against oxidative injury. Eur J Pharmacol 580: 12-18, 2008.

23. Glotin AL, Calipel A, Brossas JY, Faussat AM, Treton J and Mascarelli F: Sustained versus transient ERK1/2 signaling underlies the anti- and proapoptotic effects of oxidative stress in human RPE cells. Invest Ophthalmol Vis Sci 47: 4614-4623, 2006.

24. Murakami T, Takagi H, Suzuma K, et al: Angiopoietin-1 attenuates $\mathrm{H} 2 \mathrm{O} 2$-induced SEK1/JNK phosphorylation through the phosphatidylinositol 3-kinase/Akt pathway in vascular endothelial cells. J Biol Chem 280: 31841-31849, 2005.
25. Zhuang S and Schnellmann RG: A death-promoting role for extracellular signal-regulated kinase. J Pharmacol Exp Ther 319: 991-997, 2006.

26. Jimenez LA, Zanella C, Fung H, et al: Role of extracellular signal-regulated protein kinases in apoptosis by asbestos and H2O2. Am J Physiol 273: L1029-L1035, 1997.

27. Li SY, Gomelsky M, Duan J, et al: Overexpression of aldehyde dehydrogenase-2 (ALDH2) transgene prevents acetaldehydeinduced cell injury in human umbilical vein endothelial cells: role of ERK and p38 mitogen-activated protein kinase. J Biol Chem 279: 11244-11252, 2004.

28. Kim YK, Kim HJ, Kwon CH, et al: Role of ERK activation in cisplatin-induced apoptosis in OK renal epithelial cells. J Appl Toxicol 25: 374-382, 2005.

29. Liu CL, Xie LX, Li M, Durairajan SS, Goto S and Huang JD: Salvianolic acid B inhibits hydrogen peroxide-induced endothelial cell apoptosis through regulating PI3K/Akt signaling. PLoS One 2: e1321, 2007

30. Datta SR, Dudek H, Tao X, et al: Akt phosphorylation of BAD couples survival signals to the cell-intrinsic death machinery. Cell 91: 231-241, 1997.

31. Kim D and Chung J: Akt: versatile mediator of cell survival and beyond. J Biochem Mol Biol 35: 106-115, 2002.

32. Blair LA, Bence-Hanulec KK, Mehta S, Franke T, Kaplan D and Marshall J: Akt-dependent potentiation of $\mathrm{L}$ channels by insulin-like growth factor-1 is required for neuronal survival. J Neurosci 19: 1940-1951, 1999.

33. Dudek H, Datta SR, Franke TF, et al: Regulation of neuronal survival by the serine-threonine protein kinase Akt. Science 275: 661-665, 1997.

34. Chen RH, Su YH, Chuang RL and Chang TY: Suppression of transforming growth factor-beta-induced apoptosis through a phosphatidylinositol 3-kinase/Akt-dependent pathway. Oncogene 17: 1959-1968, 1998. 